\title{
Inertial Vibration Control Using a Shunted Electromagnetic Transducer
}

\author{
Andrew J. Fleming, Member, IEEE, and S. O. Reza Moheimani, Senior Member, IEEE
}

\begin{abstract}
Inertial drives and passive tuned-mass dampers utilize a suspended mass to reduce the vibration experienced by a host structure. Active vibration control systems typically include a voice coil type electromagnetic actuator to develop the required reaction forces. In this paper, the technique of sensorless active shunt control is applied to inertial vibration absorption. An electrical impedance is designed and connected to an electromagnetic coil with a view to minimizing structural vibration. Standard optimal control tools can be applied to design the required shunt impedance. This technique requires no additional feedback sensors. Vibration in an experimental structure is heavily attenuated by the application of an active shunt impedance.
\end{abstract}

Index Terms-Control, electromagnetic, inertial, proof mass, senorless, shunt damping, vibration.

\section{INTRODUCTION}

$\mathbf{T}$ UNED mass dampers, or inertial drives, are commonly used for mechanical vibration control [1], [2]. Passive tuned mass absorbers utilize an inertial mass and tuned support to introduce additional dynamics and mitigate vibration over a certain frequency range. Inertial drives, comprising an electromagnetic transducer and suspended inertial mass, are used in active feedback control systems to regulate measured acceleration.

Electromagnetic transducers can be used as sensors, actuators, or both [3]-[8]. A simple technique for mitigation of mechanical vibration is known as passive electromagnetic shunt damping [9]. Analogous to the well-known technique of piezoelectric shunt damping [10], an electrical impedance, designed to reduced vibration, is connected to the terminals of an electromagnetic transducer. A great variety of piezoelectric shunt circuit designs, reviewed in [11], have been proposed for reducing structural vibration. A subset of these techniques has been adapted for use with electromagnetic transducers [9]. Unfortunately, such techniques find limited application, as the control objective offered by piezoelectric shunt damping circuits is not valid in the context of electromagnetic vibration control. Piezoelectric shunt damping circuits result in the reduction of strain under the transducer [11]; analogously, their adapted electromagnetic equivalents mitigate the relative velocity experienced

Manuscript received December 15, 2003; revised June 3, 2005. Recommended by Technical Editor R. Rajamani. This work was supported in part by the Australian Research Council and in part by the Center for Complex Dynamic Systems and Control.

The authors are with the School of Electrical Engineering and Computer Science, University of Newcastle, Callaghan, NSW 2308, Australia (e-mail: Andrew.Fleming@newcastle.edu.au; Reza.Moheimani@newcastle.edu.au).

Digital Object Identifier 10.1109/TMECH.2005.863364 by the coil and magnetic field [9]. Practical vibration control applications utilizing electromagnetic transducers can be categorized almost universally as: inertial mass systems, isolation systems, or suspension systems. In each application, minimizing the relative velocity experienced by the transducer is contradictory to the respective performance objective. For this reason, previous results in the field of smart structures and piezoelectric shunt damping cannot be applied directly.

A technique for the synthesis of active electromagnetic shunt impedances was presented in [12]. Using active synthesis, performance objectives other than minimization of the relative velocity are possible. Active electromagnetic shunt control is potentially applicable to vibration damping, isolation systems, and suspension systems.

This paper considers vibration reduction using an electromagnetically actuated inertial drive. Both the passive and active drive dynamics are considered when constructing a model of the mated mechanical and inertial drive systems. In doing so, the performance of a passive absorber can be augmented with an active feedback system. A method is presented for the modeling, design, and implementation of a shunt controlled electromagnetic inertial drive for vibration suppression. By viewing the coil current and voltage as system inputs and outputs, the task of impedance synthesis can be cast as a standard feedback design problem. Arbitrary objectives such as $L Q R, L Q G$, or $\mathcal{H}_{2}$ goals are easily specified. In this work, displacement is minimized subject to a penalty on the inertial mass travel and applied terminal voltage. Using this technique, the need for external sensors is eliminated, significantly reducing the cost, complexity, and sensitivity to transducer failure that in many applications may preclude the use of an active control system.

Experiments are performed on a simple single-mode host structure with integrated electromagnetic transducer and suspended absorber mass. The combination of passive and active control results in significant vibration suppression.

This paper is organized as follows. In Section II, we begin with the modeling of mechanical and electromagnetic systems. A method is then presented in Section III for the design of active impedance and admittance controllers to minimize a specified performance objective. The presented techniques are then applied to an experimental electromagnetic system in Section IV. Finally, the paper is concluded in Section V.

\section{MODELING}

In this section, the dynamics of an electromagnetic and mechanical system are studied independently, then combined to reveal the dynamics of a shunted electromagnetic inertial actuator. 


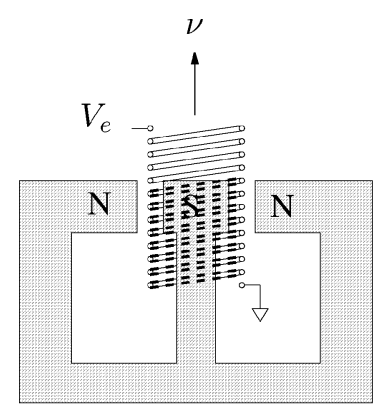

(a)

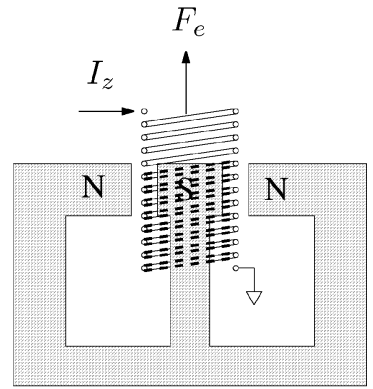

(b)
Fig. 1. A voice-coil-type electromagnetic transducer in (a) a velocity sensing role and (b) force actuating role.

\section{A. Electromagnetic System}

When an electrical conductor, in the form of a coil, moves in a magnetic field as shown in Fig. 1(a), a voltage $V_{e}$ proportional to the velocity $\nu$ is induced and appears across the terminals of the coil

$$
V_{e} \propto \nu
$$

Specifically,

$$
\frac{V_{e}}{\nu}=B l
$$

where $B$ is the magnetic flux (in T), $l$ is the length of the conductor (in $\mathrm{m}$ ), and $\nu$ is the velocity of the conductor relative to the magnetic field (in $\mathrm{ms}^{-1}$ ). A permanent magnet is usually the source of the magnetic field. In another configuration, the coil is kept stationary and the magnet is free to move.

Assuming the coil is exposed to a field of constant flux density and the relative displacement is small, (2) can be rewritten [1] as

$$
\frac{V_{e}}{\nu}=\frac{F_{e}}{I_{z}}=B l=C_{e}
$$

where $F_{e}$ denotes the force (in newtons) acting on the coil carrying a current $I_{z}$ (in amperes), and $C_{e}$ is the ideal electromechanical coupling coefficient. When the coil is employed as a force actuator, (3) relates the induced force to an applied current. Electrodynamic shakers and acoustic speakers operate on this principle. As shown in Fig. 2, the coil can be modeled as the series connection of an inductor $L$, a resistor $R$, and a dependent voltage source $V_{e}$ [4]. When coupled to a mechanical system, the induced electromotive force (EMF) and, hence, mechanical velocity, can be determined from the open circuit coil terminal voltage. The electrical and mechanical transducer dynamics are summarized in Fig. 3. In response to a relative velocity $\nu$ and terminal voltage $V_{z}$, the transducer $E$ develops a force $F_{e}$ and current $I_{z}$. When short circuited; i.e., when $V_{z}=0$, the transducer develops a force opposite in direction to the relative coil velocity $\nu$. This damping force is utilized in electrodynamic braking systems and eddy-current dampers. (a)

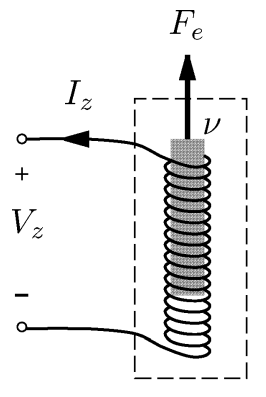

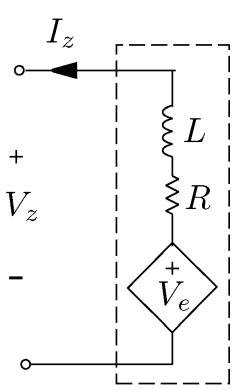

(b)
Fig. 2. (a) An electromagnetic transducer generating a force $F_{e}$ in response to the terminal current $I_{z}$. (b) The electrical equivalent model includes the coil inductance $L$, the coil resistance $R$, and a voltage source $V_{e}$ proportional to the velocity between the coil and magnetic field.

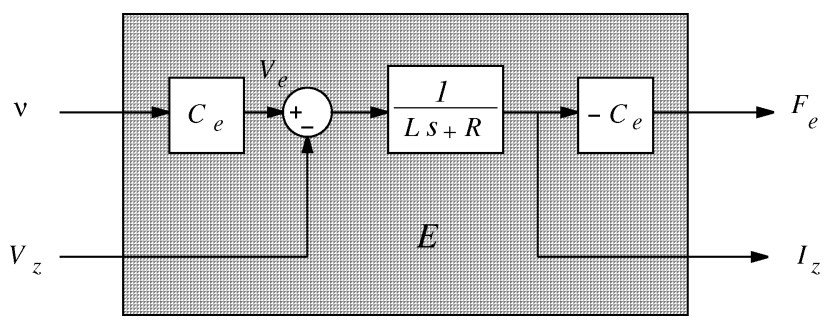

Fig. 3. The electrical and mechanical dynamics of an electromagnetic transducer.

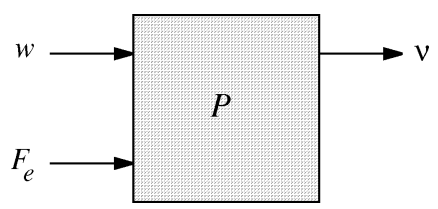

Fig. 4. A general mechanical system suitable for couping to an electromagnetic transducer. In addition to a disturbance input $w$, the model also includes a force input $F_{e}$ and relative velocity output $\nu$.

The following state-space representation of the coil admittance $Y_{c}(s)=\frac{1}{L s+R}$ will be required as

$$
A_{y}=\left[\frac{-R}{L}\right] \quad B_{y}=[1] \quad C_{y}=\left[\frac{1}{L}\right] .
$$

\section{B. Mechanical System}

The general model of a mechanical system is shown in Fig. 4. In addition to various application-specific inputs and outputs, in order to couple the system to an electromagnetic actuator, the model requires a force input $F_{e}$ and a relative velocity output $\nu$. In a typical scenario, the model would also describe the influence of a specific disturbance input $w$. A single degreeof-freedom tuned-mass, or inertial vibration control system, is shown in Fig. 5. The reaction force $F_{e}$ generated by an electromagnetic actuator is employed to minimize the vibration $d_{2}$ resulting from a disturbance force $F_{d}$. The quantities denoted $M, k, b$, and $d$ represent the mass (in $\mathrm{kg}$ ), the spring constant (in $\mathrm{N} \cdot \mathrm{m}^{-1}$ ), the damping coefficient (in $\mathrm{N} \cdot \mathrm{s} \cdot \mathrm{m}^{-1}$ ), and the displacement (in meters), respectively. The symbols $\dot{\nu}$ and $\nu$ are used to represent acceleration and velocity, respectively. 


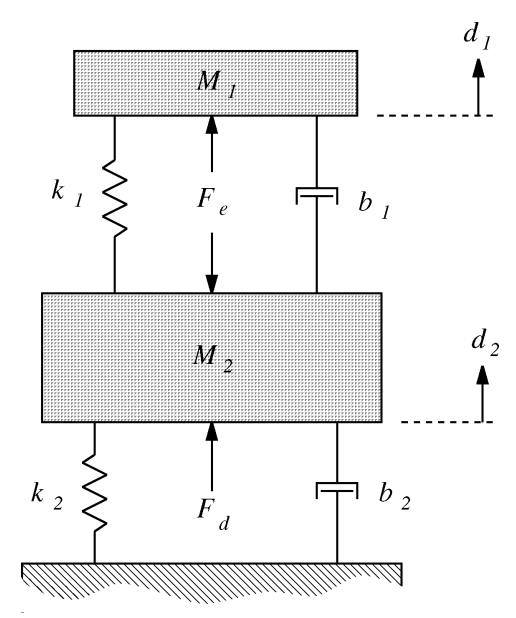

Fig. 5. A suspended mass $M_{2}$ disturbed by a force $F_{d}$. The absorber mass $M_{1}$ is maneuvered by the reaction force $F_{e}$ to reduce the displacement $d_{2}$.

The equations of motion governing the system can be written as

$$
\begin{aligned}
M_{1} \dot{\nu}_{1}= & -b_{1}\left(\nu_{1}-\nu_{2}\right)-k_{1}\left(d_{1}-d_{2}\right)+F_{e} \\
M_{2} \dot{\nu}_{2}= & b_{1}\left(\nu_{1}-\nu_{2}\right)+k_{1}\left(d_{1}-d_{2}\right)-b_{2} \nu_{2} \\
& -k_{2} d_{2}-F_{e}+F_{d} .
\end{aligned}
$$

By choosing the state variables $\nu_{1}, d_{1}, \nu_{2}$, and $d_{2}$, the system can be cast in the following state-space form:

$$
\begin{aligned}
\dot{x}_{p}(t) & =\mathbf{A}_{p} x_{p}(t)+\mathbf{B}_{p}\left[\begin{array}{c}
F_{d}(t) \\
F_{e}(t)
\end{array}\right] \\
{\left[\begin{array}{c}
d_{2}(t) \\
\nu_{1}-\nu_{2}
\end{array}\right] } & =\mathbf{C}_{p} x_{p}(t)
\end{aligned}
$$

where the subscript $p$ denotes the mechanical plant, and the output $\nu_{1}-\nu_{2}$ represents the relative velocity between the two masses. The system matrices are

$$
\begin{aligned}
x_{p}(t) & =\left[\begin{array}{l}
\nu_{1} \\
d_{1} \\
\nu_{2} \\
d_{2}
\end{array}\right] \\
\mathbf{B}_{p} & =\left[\mathbf{B}_{p 1} \mathbf{B}_{p 2}\right]=\left[\begin{array}{cc}
0 & \frac{1}{M_{1}} \\
0 & 0 \\
\frac{-1}{M_{2}} & \frac{1}{M_{2}} \\
0 & 0
\end{array}\right] \\
\mathbf{A}_{p} & =\left[\begin{array}{cccc}
\frac{-b_{1}}{M_{1}} & \frac{-k_{1}}{M_{1}} & \frac{b_{1}}{M_{1}} & \frac{k_{1}}{M_{1}} \\
1 & 0 & 0 & 0 \\
\frac{b_{1}}{M_{2}} & \frac{k_{1}}{M_{2}} & \frac{-b_{1}-b_{2}}{M_{2}} & \frac{-k_{1}-k_{2}}{M_{2}} \\
0 & 0 & 1 & 0
\end{array}\right] \\
\mathbf{C}_{p} & =\left[\begin{array}{cccc}
\mathbf{C}_{p 1} \\
\mathbf{C}_{p 2}
\end{array}\right]=\left[\begin{array}{cccc}
0 & 0 & 0 & 1 \\
1 & 0 & -1 & 0
\end{array}\right] .
\end{aligned}
$$

A block diagram of the mechanical system (7) is shown in Fig. 6. As the system includes a control force input $F_{e}$ and a relative velocity output $\nu$, the model is easily coupled to that of an electromagnetic transducer.

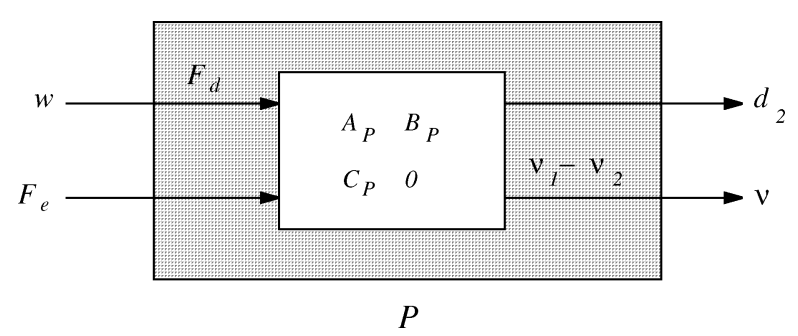

Fig. 6. The mechanical system (7) shown with force disturbance and control inputs $F_{d}$ and $F_{e}$, a performance output $d_{2}$, and the relative velocity output $\nu$.

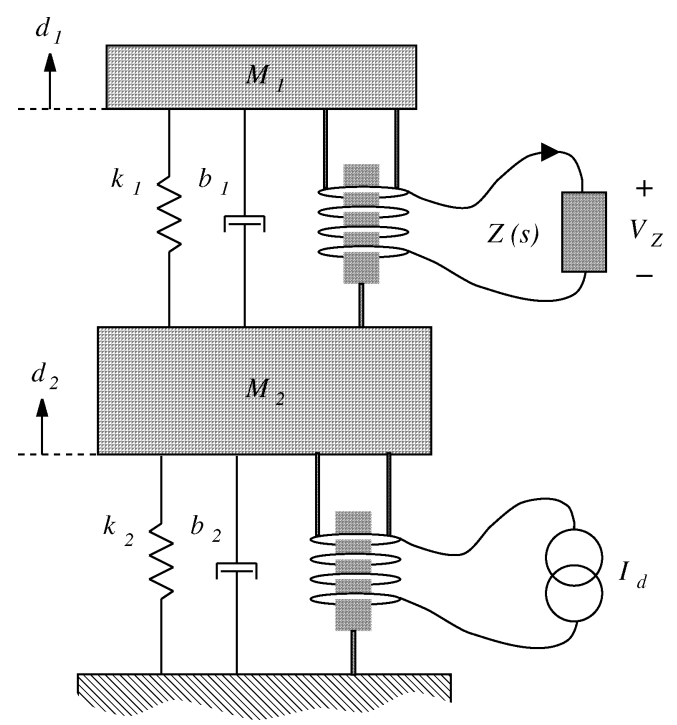

Fig. 7. A two-mass system with electromagnetic transducers to realize the disturbance force $F_{d}$ and control force $F_{e}$.

\section{Shunted Composite Electromechanical System}

As shown in Fig. 7, we now consider a mechanical system $P$ coupled to an impedance shunted electromagnetic transducer. The force disturbance $F_{d}$ is realized with the use of an auxiliary transducer and current source

$$
F_{d}=I_{d} C_{e}
$$

Within the modeling framework introduced in Sections II-A and II-B, i.e., by treating the mechanical plant and shunted electromagnetic coil as shown in Figs. 6 and 3, the composite plant is easily constructed as that shown in Fig. 8. In Fig. 8, the impedance $Z(s)$, appears analogous to a feedback controller for the electromechanical system. By concatenating the mechanical and electromagnetic systems, $P$ and $E$, as shown in Fig. 9, the composite system is cast as a typical regulation problem for the abstracted system $G$. The state equation of the electrical (4) and mechanical systems (7) can be collected to describe the system $G$

$$
\begin{aligned}
\dot{x}_{g}(t) & =\mathbf{A}_{g} x_{g}(t)+\mathbf{B}_{g}\left[\begin{array}{c}
I_{d}(t) \\
V_{z}(t)
\end{array}\right] \\
{\left[\begin{array}{c}
d_{2}(t) \\
I_{z}(t)
\end{array}\right] } & =\mathbf{C}_{g} x_{g}(t)
\end{aligned}
$$




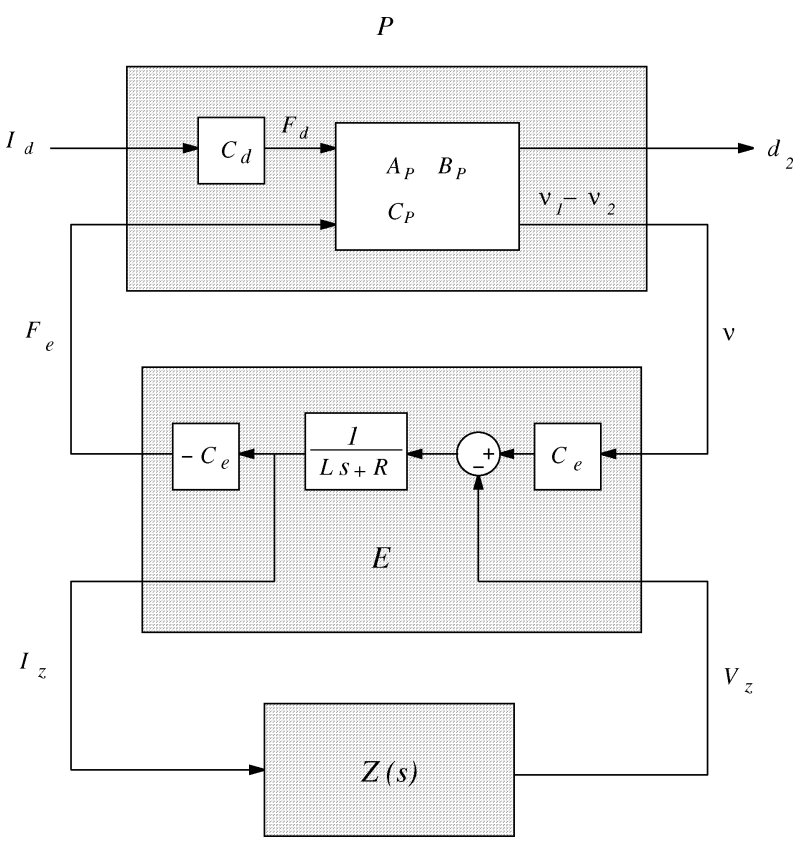

Fig. 8. Mechanical system $P$ coupled to an impedance shunted electromagnetic transducer $E$.

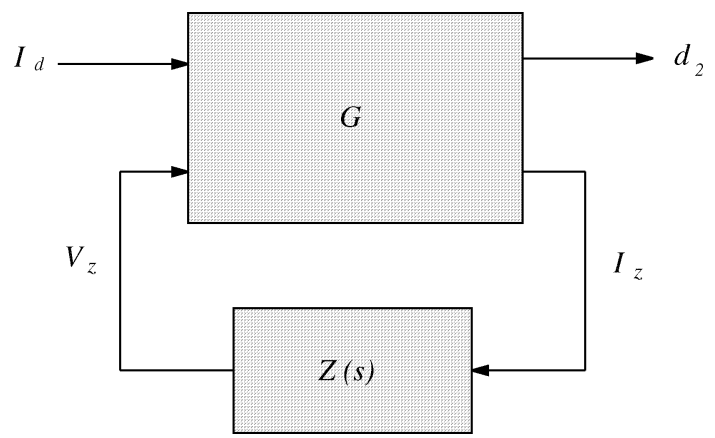

Fig. 9. Composite system $G$ comprising the mechanical and electromagnetic subsystems.

where

$$
\begin{aligned}
@_{g}(t) & =\left[\begin{array}{l}
x_{p}(t) \\
x_{y}(t)
\end{array}\right] \\
\mathbf{A}_{g} & =\left[\begin{array}{cc}
\mathbf{A}_{p} & -C_{e} \mathbf{B}_{p 2} \mathbf{C}_{y} \\
B_{y} \mathbf{C}_{p 2} C_{e} & A_{y}
\end{array}\right]
\end{aligned}
$$

and

$$
\mathbf{B}_{g}=\left[\begin{array}{cc}
\mathbf{B}_{p 1} C_{d} & 0 \\
0 & -B_{y}
\end{array}\right], \quad \mathbf{C}_{g}=\left[\begin{array}{cc}
\mathbf{C}_{p 1} & 0 \\
0 & C_{y}
\end{array}\right] .
$$

\section{Control Design}

As shown in Fig. 8, an impedance connected to a mechanically coupled electromagnetic transducer can be viewed as parameterizing a velocity feedback controller for the mechanical system $P$. Section III-A introduces a technique for the synthesis of active impedance controllers designed to minimize structural vibration.
Our design objective is to minimize the displacement $d_{2}$ while restraining both the magnitude of control voltage $V_{z}$ and the absorber mass travel $d_{1}-d_{2}$. As the reaction force $F_{e}$ results in an acceleration of the absorber, at low frequencies, the magnitude of available force is strictly limited by the maximum travel $d_{1}-d_{2}$. In a linear quadratic sense, our objective is to minimize

$$
J=\int_{0}^{\infty}\left\{d_{2}^{2}(t)+k_{v} V_{z}^{2}(t)+k_{d}\left(d_{1}(t)-d_{2}(t)\right)^{2}\right\} d t .
$$

where $k_{v}$ and $k_{d}$ represent weightings on the applied shunt voltage $V_{z}$ and the absorber mass travel $d_{1}-d_{2}$, respectively. By substituting the state solutions into $d_{1}(t)$ and $d_{2}(t)$, we obtain

$$
J=\int_{0}^{\infty}\left\{\begin{array}{c}
x_{p}^{\prime}(t) b f C_{p 1}^{\prime} \mathbf{C}_{p 1} x_{p}(t)+V_{z}(t)^{\prime} k_{v} V_{z}(t) \\
+x_{p}^{\prime}(t) \mathbf{C}_{p 3}^{\prime} k_{d} \mathbf{C}_{p 3} x_{p}(t)
\end{array}\right\} d t .
$$

where $d_{1}(t)-d_{2}(t)=\mathbf{C}_{p 3} x_{p}(t)$, and $\mathbf{C}_{p 3}=\left[\begin{array}{llll}0 & -1 & 0 & 1\end{array}\right]$. Restated in the standard $L Q R$ context,

$$
J=\int_{0}^{\infty}\left\{x_{g}^{\prime}(t) \mathbf{Q} x_{g}(t)+u(t)^{\prime} \mathbf{R} u(t)\right\} d t
$$

where

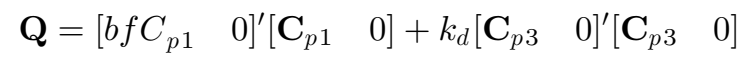

and

$$
\mathbf{R}=k_{v} .
$$

Through the solution of an algebraic Ricatti equation [13], a state feedback matrix $K$ can be found that minimizes the objective function $J$.

\section{A. Observer Design}

As the state variables of the system $x_{g}(t)$ are not available directly, a linear observer is required.

For impedance design, the ad hoc pole placement approach to linear observer design becomes difficult. Although an $L Q R$ state-feedback regulator is guaranteed (if $\mathbf{R}$ is chosen diagonal) to result in a phase margin of at least $60^{\circ}$ at each plant input channel [14], [15], it is well known that considerable degradation of the stability margins is to be expected after inclusion of the observer dynamics. A more automated choice in observer design is the Kalman filter [16]. Here, as shown in Fig. 10, the controller $C(s)$ consists of an optimal state-feedback regulator $K$ and Kalman observer $O$. By the Certainty Equivalence Principle or Separation Theorem [13], the two entities can be designed independently. After first finding a $K$ to minimize (17), we then design a Kalman filter to minimize

$$
J_{k}=\lim _{t \rightarrow \infty} E\left\{[x(t)-\tilde{x}(t)][x(t)-\tilde{x}(t)]^{\prime}\right\} .
$$

By the Certainty Equivalence Principle, the optimal $K$ and $O$ also result in minimization of the stochastic performance objective

$$
J=E\left\{\lim _{T \rightarrow \infty} \frac{1}{T} \int_{0}^{T}\left\{x^{\prime}(t) \mathbf{Q} x(t)+u(t)^{\prime} \mathbf{R} u(t)\right\} d t\right\} .
$$




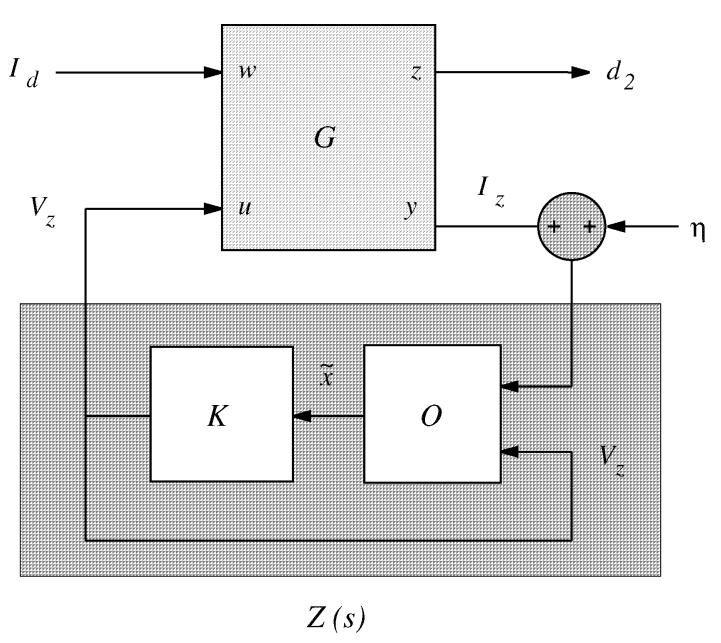

Fig. 10. Composite plant $G$ controlled by $Z(s)$, an impedance consisting of the optimal state-feedback regulator $K$ and Kalman filter $O$.

In this scenario, we are referring to the original state-space system (12) with zero-mean uncorrelated Gaussian process models for the disturbance $I_{d}$ and additive measurement noise $\eta$. With the inclusion of measurement noise, the system representation (12) becomes

$$
\begin{gathered}
\dot{x}_{g}=\mathbf{A}_{g} x_{g}+\mathbf{B}_{g}\left[\begin{array}{c}
I_{d} \\
V_{z}
\end{array}\right] \\
{\left[\begin{array}{c}
d_{2} \\
I_{z}
\end{array}\right]=\mathbf{C}_{g} x_{g}+\left[\begin{array}{l}
0 \\
\eta
\end{array}\right]}
\end{gathered}
$$

where $I_{d}$ and $\eta$ satisfy

$$
\begin{aligned}
E\left\{I_{d} I_{d}^{\prime}\right\} & =\mathbf{Q}_{n} \\
E\left\{\eta \eta^{\prime}\right\} & =\mathbf{R}_{n} .
\end{aligned}
$$

Based on $\mathbf{Q}_{n}$ and $\mathbf{R}_{n}$, a Kalman observer that minimizes (20) can be found through the solution of an algebraic Ricatti equation [13]. The ratio of $\mathbf{Q}_{n}$ to $\mathbf{R}_{n}$ essentially represents the confidence in the measured variable $I_{z}$ and plant model $G$. In this work, $\mathbf{Q}_{n}, \mathbf{R}_{n}$, and $\mathbf{k}_{u}$ are not quantified and are simply treated as design parameters influencing the closed-loop pole locations, damping performance, and closed-loop stability.

\section{EXPERIMENTAL RESULTS}

To verify the modeling and design techniques presented in the preceding sections, each method has been applied to an experimental electromechanical system.

\section{A. Experimental Apparatus}

A photograph of the experimental apparatus showing the rigid body, flexible end supports, mounting plate, and coils is provided in Fig. 11. As shown in Fig. 12, the apparatus comprises two electromagnetic transducers mounted vertically. While the lower magnet and flexure is fixed, the two connected transducer cases are free to vibrate and represent the mass $M_{2}$. The upper magnet forms the absorber mass $M_{1}$. Each transducer is essen-

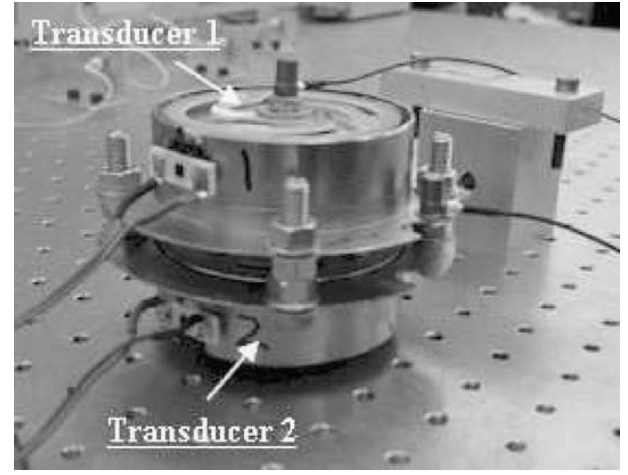

Fig. 11. Experimental apparatus.

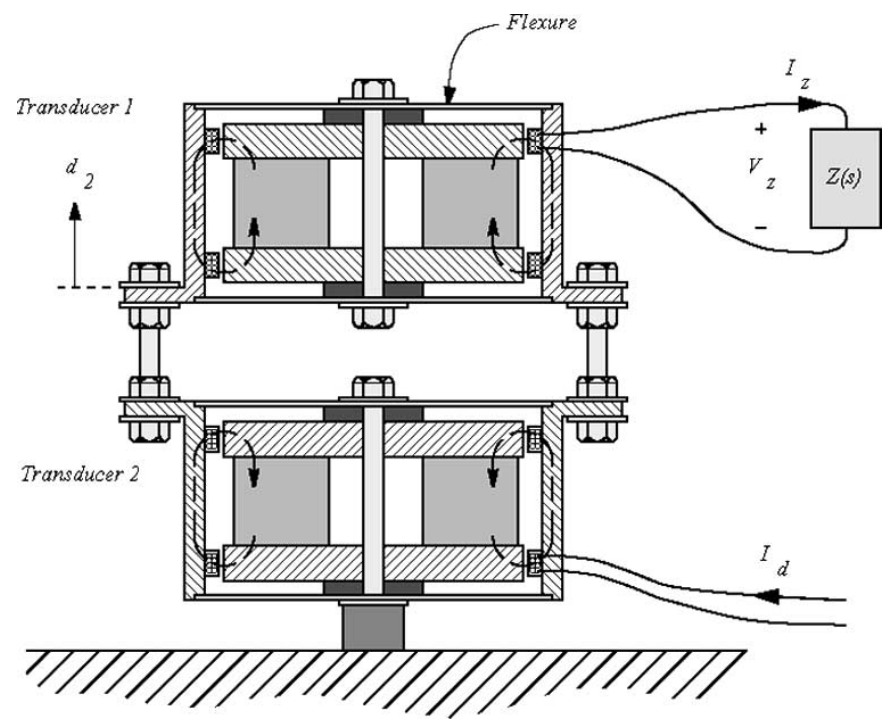

Fig. 12. Cross section of the experiment apparatus shown in Fig. 11.

TABLE I

ELECTROMECHANICAL SYSTEM PARAMETERS

\begin{tabular}{|l|l|}
\hline Parameter & Value \\
\hline Spring constant $k_{1}$ & $28.03 \mathrm{kNm}^{-1}$ \\
\hline Damping coefficient $b_{1}$ & $1.500 \mathrm{Nsm}^{-1}$ \\
\hline Absorber mass $M_{1}$ & $0.340 \mathrm{~kg}$ \\
\hline Spring constant $k_{2}$ & $31.14 \mathrm{kNm}^{-1}$ \\
\hline Damping coefficient $b_{2}$ & $3.582 \mathrm{Nsm}^{-1}$ \\
\hline Absorber mass $M_{2}$ & $0.593 \mathrm{~kg}$ \\
\hline Coil inductance & $41 \mathrm{mH}$ \\
\hline Coil resistance & $2.315 \Omega$ \\
\hline Electromagnetic Coupling $C_{e}$ & 3.408 \\
\hline Electromagnetic Coupling $C_{d}$ & -6.714 \\
\hline
\end{tabular}

tially a translational solenoid with two identical coils connected in series; the dashed line identifies the flux path generated by the permanent magnets. The physical parameters of the electromagnetic and mechanical systems are summarized in Table I.

The main mass velocity $d_{2}$ is measured using a PSV-300 Polytec Scanning Laser Vibrometer.

\section{B. Power Amplifier and Instrumentation}

In order to implement an arbitrary shunt impedance, a power amplifier was developed capable of driving differential terminal 


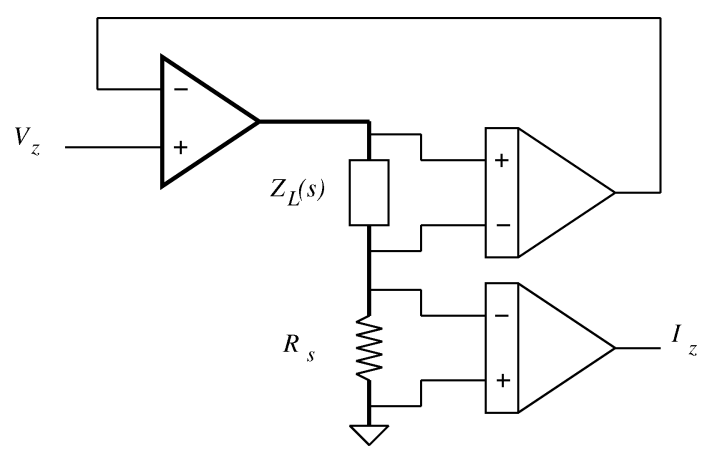

Fig. 13. Simplified schematic of a voltage source with current instrumentation. The load impedance $Z_{L}(s)$ represents the electromagnetic coil.

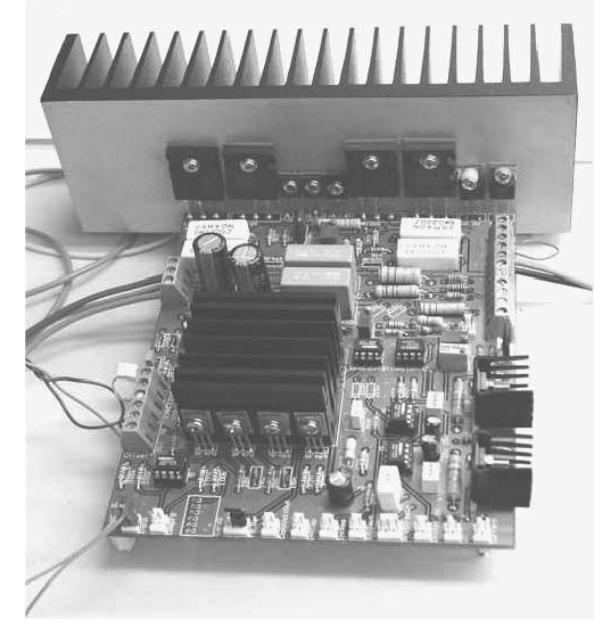

Fig. 14. Practical implementation of a voltage amplifier with current instrumentation.

voltages with load current instrumentation. The simplified schematic of a circuit realizing this function is shown in Fig. 13. A practical implementation of the amplifier is shown in Fig. 14. The device is capable of $\pm 200 \mathrm{~V}$ operation at a maximum dc current of $32 \mathrm{~A}$. Further analysis and a more detailed discussion of the implementation can be found in [17]. A dSpace 1005 based system is used to implement the required impedance and admittance transfer functions.

\section{Impedance Synthesis}

Fig. 15 shows the instrumentation and driver gains associated with the underlying electromechanical system. The voltages $V_{1}-V_{4}$ represent the signals applied to, or measured from, the power amplifiers and instrumentation, respectively. The gain and units associated with each signal can be found in Table II. The actual electrical shunt impedance presented to the coil is related to the controller through the gains $a_{3}$ and $a_{4}$; specifically,

$$
Z(s)=\frac{V_{z}(s)}{I_{z}(s)}=a_{3} C(s) a_{4} .
$$

To assess the accuracy of the analytic model (discussed in Section II-C), the simulated frequency response is compared to that measured directly from the experimental system. A multi-

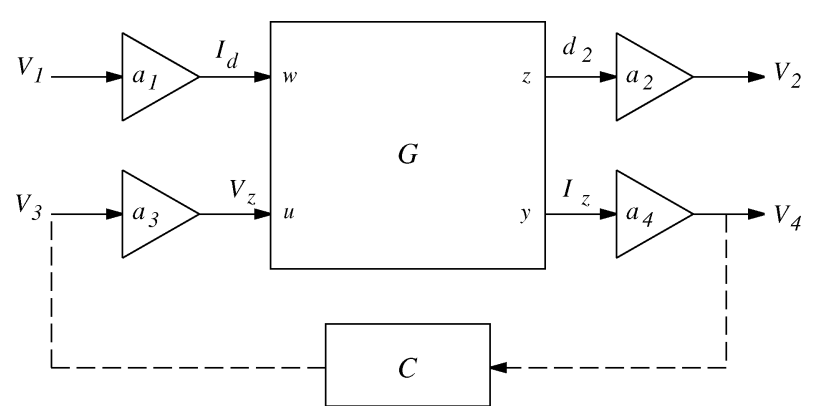

Fig. 15. External gains associated with the amplifier and instrumentation.

TABLE II

EXTERNAL GAINS ASSOCIATED WITH THE EXPERIMENTAL SYSTEM

\begin{tabular}{|l|l|}
\hline Gain & Value \\
\hline$a_{1}$ & $1.006 \mathrm{~A} / \mathrm{V}$ \\
\hline$a_{2}$ & $1 \mathrm{~V} / \mathrm{m}$ \\
\hline$a_{3}$ & $-1.012 \mathrm{~V} / \mathrm{V}$ \\
\hline$a_{4}$ & $-10.01 \mathrm{~V} / \mathrm{A}$ \\
\hline
\end{tabular}
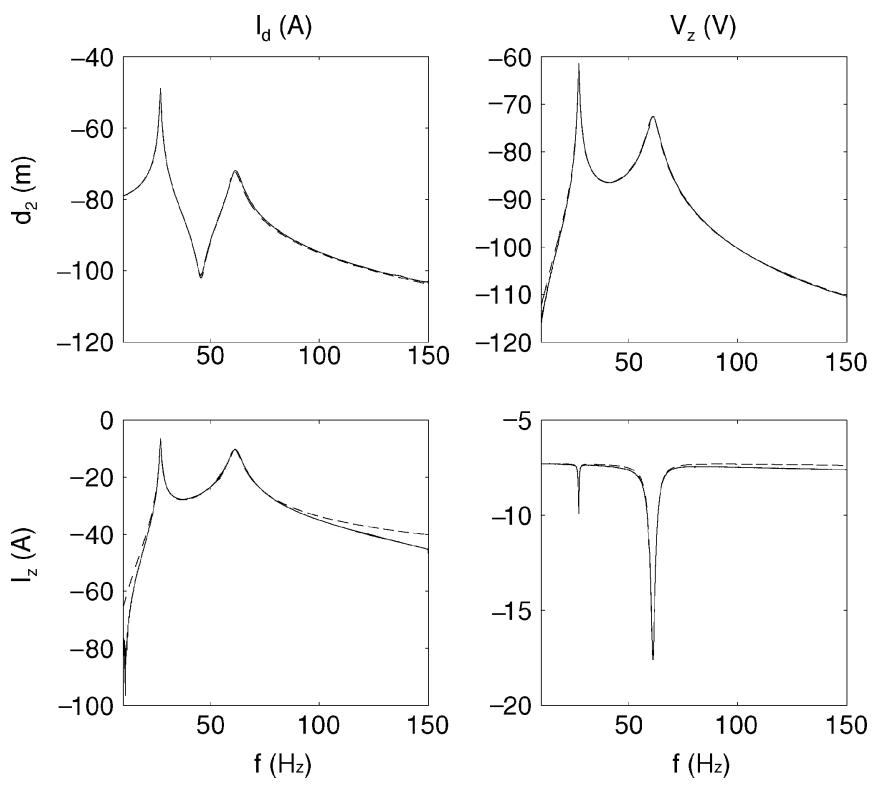

Fig. 16. Simulated (-) and experimental (- $)$ magnitude frequency response.

variable frequency response is measured successively from each input to output pair. During the component SISO frequency response measurements, the residual input is set to zero. The magnitude and phase frequency responses are shown, respectively, in Figs. 16 and 17. In the frequency domain, a good correlation can be observed between the analytic model and measured system.

1) LQR Impedance Synthesis: As discussed in Section III, a linear quadratic regulator can be designed to command the shunt terminal voltage $V_{z}$ with a view to minimizing the vibration $d_{2}$. An observer is required to estimate the system states from the measured shunt current $I_{z}$.

Based on the physical model that was validated perviously, and referring to the notation introduced in Section III, an $L Q R$ 

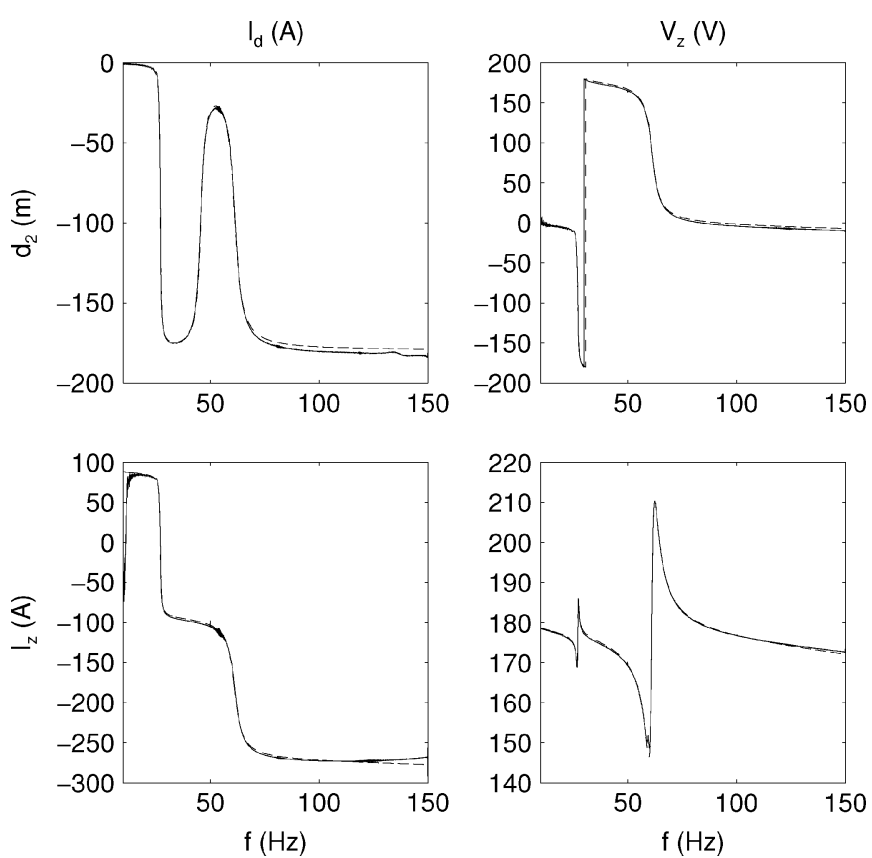

Fig. 17. Simulated (-) and experimental (- -) phase frequency response.
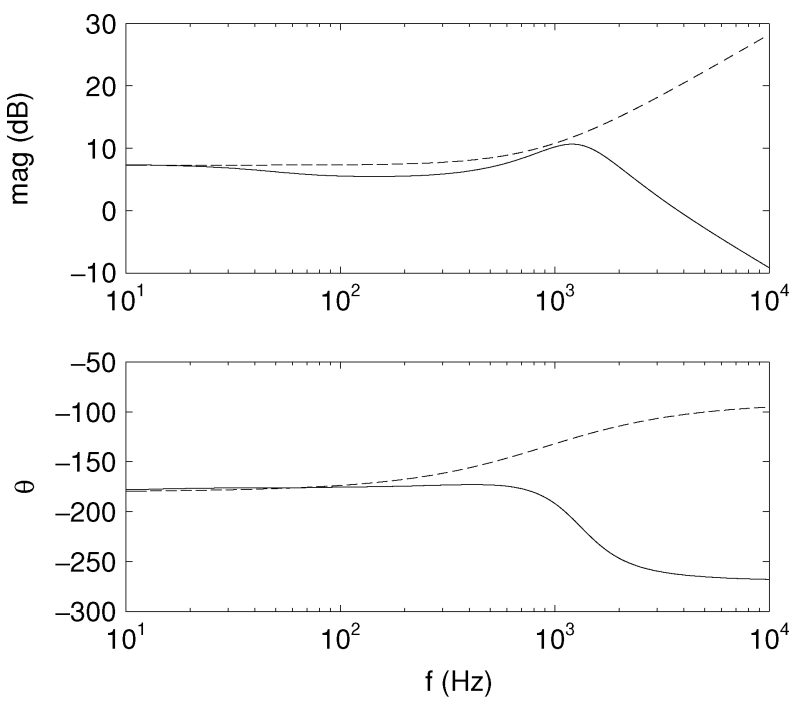

Fig. 18. Magnitude and phase response of the LQG impedance (-) and negative coil impedance (- -).

gain matrix was designed to minimize the performance function

$$
J=\int_{-\infty}^{\infty}\left\{d_{2}^{2}(t)+k_{v} V_{z}^{2}(t)+k_{d}\left(d_{1}(t)-d_{2}(t)\right)^{2}\right\} d t
$$

where the factors $k_{d}=1$ and $k_{v}=1 \times 10^{-7}$. A Kalman observer was designed to estimate the system state $x_{g}(t)$ utilizing the measured shunt transducer current $I_{z}$ and control signal $V_{z}$. Referring to Section III-A, the disturbance and output noise process covariance matrices $\mathbf{Q}_{n}$ and $\mathbf{R}_{n}$ were chosen to be 100 and 0.1 , respectively. Such a weighting, although not quantitative, expresses a moderate confidence in the fidelity of the measured variable $I_{z}$.

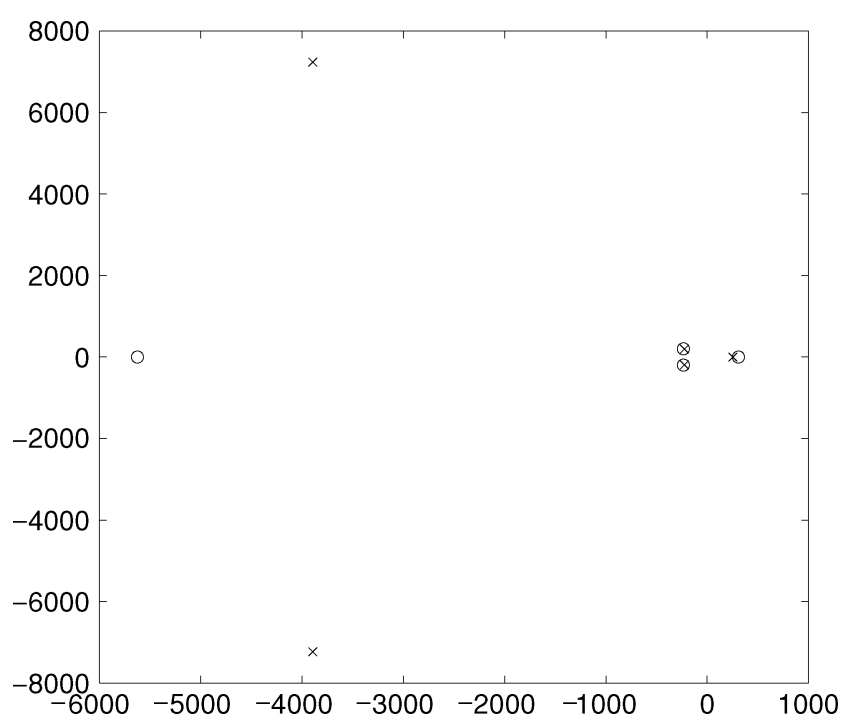

Fig. 19. Pole-zero map of the LQG impedance.

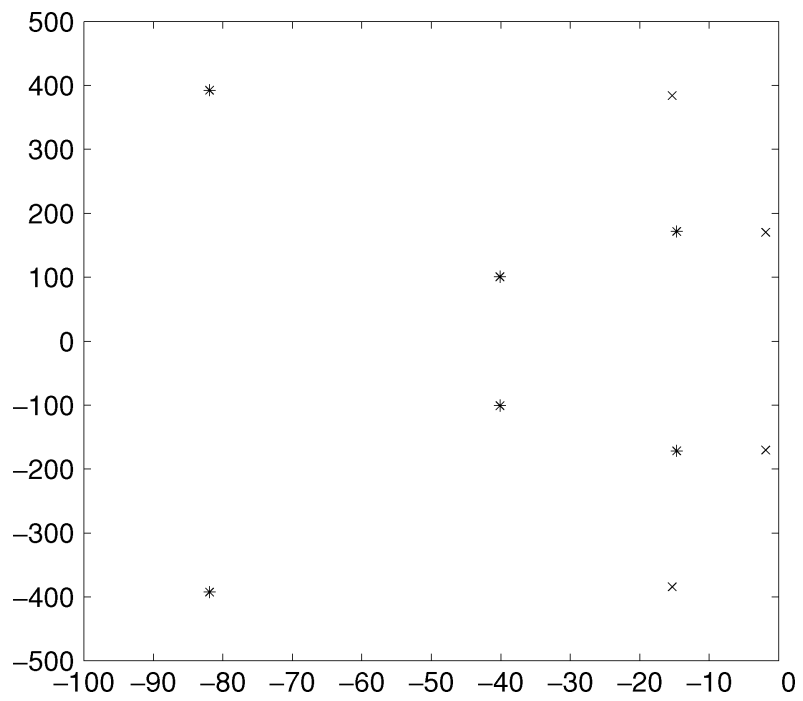

Fig. 20. Open $(x)$ and shunted $(\star)$ pole locations. Note that one pair of high-frequency observer poles are not visible within the scope of this plot.

By concatenating the $L Q R$ gain matrix and Kalman observer, and compensating for the system gains $a_{3}$ and $a_{4}$, the actual impedance presented to the shunt transducer can be determined. In Fig. 18, the complex impedance of the resulting controller is plotted together with that of the negated coil impedance. A negative coil impedance connected to the true coil impedance effectively removes the source impedance from the transducer. The $L Q G$ impedance has a tendency to mimic this impedance over a certain frequency range. The pole-zero map of the $L Q G$ controller is shown in Fig. 19.

After examining the open- and closed-loop pole locations shown in Fig. 20, it can be appreciated that the controller is clearly acting to increase the system damping. Corresponding mitigation of the transfer function from an applied disturbance to the measured vibration can be seen in both the frequency 


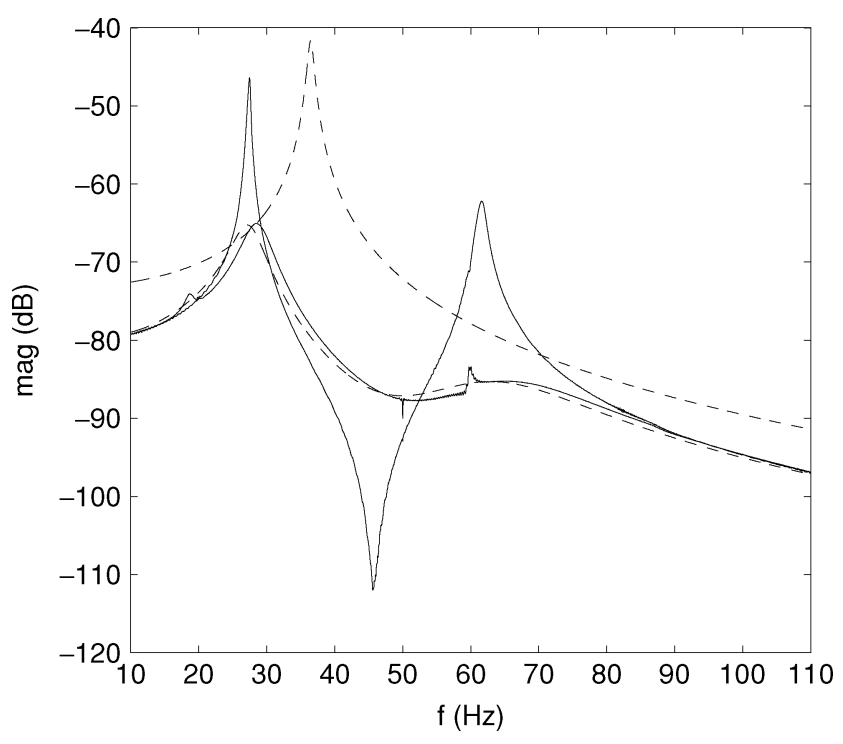

Fig. 21. Single-mass (- -), two-mass (-), experimental shunted (-), and simulated shunted (- -) magnitude frequency response $d_{2}(s) / I_{d}(s)$.

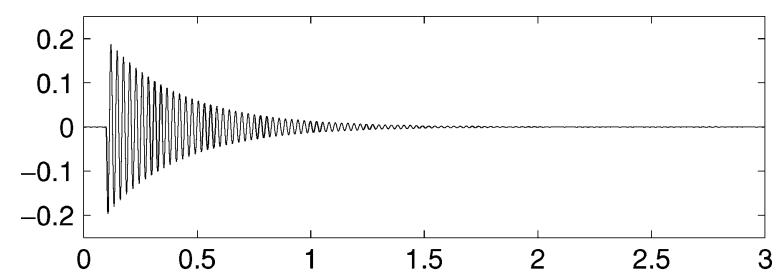

(a)

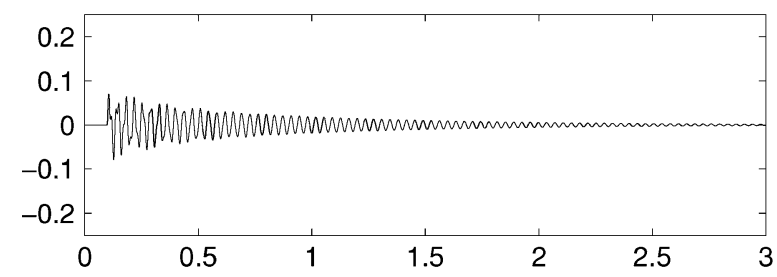

(b)

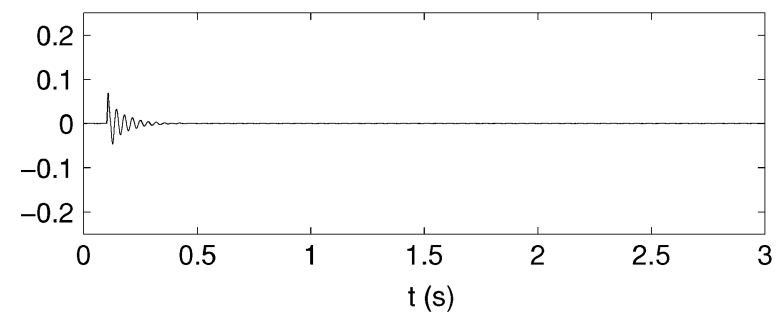

(c)

Fig. 22. Single-mass. (a) Two-mass. (b) Shunted two-mass. (c) Velocity response to a 1-A step in $I_{d}$.

domain, Fig. 21, and time domain, Fig. 22. The action of the additional mass and electromagnetic shunt acts to reduce the single-mass resonant peak by a minimum of $23.2 \mathrm{~dB}$. The shunted electromagnetic transducer reduces the two-mass first and second resonant peaks by 18.7 and $23.6 \mathrm{~dB}$, respectively.

The reader may note additional dynamics at 20 and $60 \mathrm{~Hz}$ in Fig. 21. These dynamics are due to pivot modes of the structure about the base fixture. The stiffening effect of the controller has a tendency to increase the frequency of low-profile pivot and sway modes. Such modes would be absent in a more rigidly supported inertial drive.

\section{CONCLUSION}

A technique has been presented for the control of vibration using an electromagnetically actuated inertial drive. By viewing the coil current and voltage as system inputs and outputs, standard synthesis techniques were applied to minimize displacement subject to a penalty on the inertial mass travel and applied terminal voltage. Electromagnetic shunt control requires no external sensors, and thus significantly reduces the cost, complexity, and sensitivity to transducer failure of active vibration control systems.

Experiments were performed on a simple apparatus representing a scenario where the vibration experienced by a host structure is controlled with a suspended absorber mass and electromagnetic coil. In practice, the mass of the absorber is usually limited to about one tenth the host structure. In this regard, the experiment is somewhat unrealistic, as the mass of the absorber is only slightly less than that of the host mass. The available control authority is directly related to both the size of the mass and the available travel.

After adding the absorber mass, the passive dynamics split the original resonant mode into two lightly damped secondary peaks. Then, by designing a suitable control impedance and presenting it to the terminals of the electromagnetic coil, further vibration reduction is achieved by augmenting the passive damping of the secondary modes. As the control design penalizes the absorber mass travel, which increases at low frequencies, the impedance suppresses higher frequency vibration more heavily. The combination of passive and active dynamics reduces the displacement response to a force input by up to $38 \mathrm{~dB}$ at the frequency of the original resonance.

Future work includes design for multimode host structures, coupled multidrive multidimensional systems, and restricted impedance design. The $L Q G$ impedance design contains negative reactive components and is unstable in a systems perspective. Although the connection of the coil and control impedance is stable, an inherently stable controller is desirable. It is presently unclear whether an unstable controller is necessary to result in effective vibration reduction.

\section{REFERENCES}

[1] S. S. Rao, Mechanical Vibrations, 3rd ed., Reading, MA: Addison-Wesley, 1995.

[2] D. J. Inman, Engineering Vibrations, 2nd ed., Englewood Cliffs, NJ: Prentice-Hall, 2000.

[3] S. Mirzaei, S. M. Saghaiannejad, V. Tahani, and M. Moallem, "Linear electric actuators and generators," IEEE Trans. Energy Convers., vol. 14, no. 3, pp. 712-717, Sep. 1999.

[4] B. M. Hanson, M. D. Brown, and J. Fisher, "Self sensing: Closed-loop estimation for a linear electromagnetic actuator," in Proc. IEEE American Control Conf., Arlington, VA, Jun. 2001, pp. 1650-1655.

[5] Y. B. Kim, W. G. Hwang, C. D. Kee, and H. B. Yi, "Active vibration control of suspension system using an electromagnetic damper," Proc. Inst. Mech. Eng. D, Transp. Eng., vol. 8, pp. 865-873, 2001. 
[6] J. Shaw, "Active vibration isolation by adaptive control," in Proc. IEEE Int. Conf. Control Applications, Hapuna Beach, HI, Aug. 1999, pp. 15091514.

[7] D. Vischer and H. Bleuler, "Self-sensing active magnetic levitation," IEEE Trans. Magn., vol. 29, no. 2, pp. 1276-1281, Mar. 1993.

[8] N. Morse, R. Smith, B. Paden, and J. Antaki, "Position sensed and selfsensing magnetic bearing configurations and associated robustness limitations," in Proc. IEEE Conf. Decision and Control, Tampa, FL, Dec. 1998, pp. 2599-2604.

[9] S. Behrens, A. J. Fleming, and S. O. R. Moheimani, "Electromagnetic shunt damping," in Proc. IEEE/ASME Int. Conf. Advanced Intelligent Mechatronics 2003, Kobe, Japan, pp. 1145-1150.

[10] N. W. Hagood and A. Von Flotow, "Damping of structural vibrations with piezoelectric materials and passive electrical networks," J. Sound Vib., vol. 146, no. 2, pp. 243-268, 1991.

[11] S. O. R. Moheimani, "A survey of recent innovations in vibration damping and control using shunted piezoelectric transducers," IEEE Trans. Contr. Syst. Technol., vol. 11, no. 4, pp. 482-494, Jul. 2003.

[12] A. J. Fleming, S. Behrens, and R. Moheimani, "Active $\mathcal{H}_{2}$ and $\mathcal{H}_{\infty}$ shunt control of electromagnetic transducers," in Proc. IEEE Conf. Decision and Control, Lahaina, Maui, HI, Dec. 2003, pp. 2294-2299.

[13] S. Skogestad and I. Postlethwaite, Multivariable Feedback Control. New York: Wiley, 1996

[14] R. Kalman, "When is a linear control system optimal," Trans. ASME, J. Basic Eng. D, vol. 86, pp. 51-60, 1964

[15] M. G. Safonov and M. Athans, "Gain and phase margin for multiloop LQG regulators," IEEE Trans. Autom. Control, vol. AC-22, no. 2, pp. 173-179, Apr. 1977.

[16] R. G. Brown and P. Hwang, Introduction to Random Signals and Applied Kalman Filtering. New York: Wiley, 1997.

[17] A. J. Fleming and S. O. R. Moheimani, "Improved current and charge amplifiers for driving piezoelectric loads, and issues in signal processing design for synthesis of shunt damping circuits," Intell. Mater. Syst. Structures, vol. 15, no. 2, pp. 77-92, Feb. 2004.

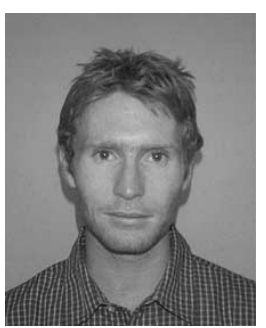

Andrew J. Fleming (M'00) was born in Dingwall, U.K., in 1977. He received the B.Eng. and Ph.D. degrees in electrical engineering from the University of Newcastle, Callaghan, Australia, in 2000 and 2004, respectively.

In 2005, he was a research academic at the Center for Complex Dynamics and Control, University of Newcastle, Australia. He is currently an Australian Research Council Fellow stationed at the School of Electrical Engineering and Computer Science, University of Newcastle, Callaghan, Australia. His research interests include micro-cantilever sensors, nano-positioning, and sensorless control of sound and vibration.

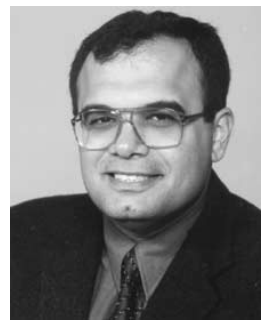

S. O. Reza Moheimani (S'93-M'97-SM'00) received the B.Sc. degree from Shiraz University, Shiraz, Iran, in 1990, and the M.Eng.Sc. and Ph.D. degrees from the University of New South Wales, Sydney, Australia, in 1993 and 1996, respectively, all in electrical and electronics engineering.

In 1996, he was a Postdoctoral Research Fellow in the School of Electrical and Electronics Engineering, Australian Defense Force Academy, Canberra, Australia. In 1997, he joined the University of Newcastle, Callaghan, Australia, where he is currently an Associate Professor in the School of Electrical Engineering and Computer Science. He has over 100 journal and conference publications, is a coauthor of the research monograph Spatial Control of Vibration: Theory and Experiments (World Scientific, 2003), and the Editor of Perspectives in Robust Control (Springer-Verlag, 2001). His research interests include smart structures, mechatronic systems, control theory, and signal processing.

Dr. Moheimani is an Associate Editor of the IEEE TRANSACTIONS ON CONTROL Systems TeChNOLOGY and International Journal of Control, Automation, and Systems, and a past Associate Editor of Control Engineering Practice. He has served on the editorial boards of several international conferences, and was the Chairman of International Program Committee for the 3rd IFAC Symposium on Mechatronic Systems held in Sydney, Australia, in September 2004. He is a Member of the ARC Center for Complex Dynamic Systems and Control, where he directs the Center's research in mechatronics. 